Original article

\title{
Prevalence of HPV infection in reproductive aged female in Delhi NCR region
}

\author{
Chandra Shekhar Kapoor ${ }^{\mathrm{a}}$, Manisha Sharma ${ }^{\mathrm{b}, *}$ \\ ${ }^{a}$ Department of Environmental Sciences, Pacific Academy of Higher Education and Research University, Udaipur, India \\ ${ }^{\mathrm{b}}$ Department of Life Science, Pacific Academy of Higher Education and Research University, Udaipur, India
}

\section{A R T I C L E I N F O}

\section{Keywords:}

Cervical cancer

Human papilloma virus (HPV)

Screening

Awareness

PAP smear

\begin{abstract}
A B S T R A C T
Purpose: The purpose of the study was to conduct screening with Pap smear cytology and HPV testing among study women. The study also aimed to further perform a correlation analyses of HPV prevalence with demographic data.

Methods: The present study collected demographic, epidemiologic and clinical information of women of aged group 20-60 years with gynaecological abnormalities who attended the gynaecology and cancer OPD at a tertiary care health center.

Results: Majority of the study participants were young married women and did not have any formal education (85.5\%). Mean (SD) age at the time of first pregnancy among the study subjects was 16.8 (2.7) years. Majority of the study participants were below poverty line (88.2\%). A total of $68.6 \%$ of the study subjects had an HPV infection. Among those that had an HPV infection, majority had HPV 16 infection (95.4\%). In the remaining 4.6\%, HPV 18 was present. Findings show that with increasing age, HPV prevalence increases. Also, early age at marriage, lack of education, increased parity, early age at first pregnancy, poor sanitation, use of tobacco and belonging to below poverty line were significant risk factors for HPV infection in this cohort.

Conclusion: The study reported a high prevalence of HPV infection in rural women attending the Gynaecology and Cancer OPD of a tertiary care hospital. Several important risk factors for the HPV infection were also identified. These were early age at marriage, lack of education, increased parity, early age at first pregnancy, poor sanitation, use of tobacco and belonging to below poverty line. These findings call for more efforts to increase awareness pertaining to HPV infection and cervical cancer among rural women.
\end{abstract}

\section{Introduction}

Cervical cancer is the fourth most common female malignancy worldwide and represents a major global health challenge. An estimated 527,600 cases and 265,700 deaths occur worldwide due to cervical cancer. ${ }^{1,2}$ In developing countries, it is the second most commonly diagnosed cancer after breast cancer and third leading cause of cancer-related death after breast and lung cancers. ${ }^{1,2}$ In India Cervical cancer incidence rate was reported 77000 (95\% CI; 68000-96000) and prevalent cases were reported 288000 (247000-342000)in year 2016. ${ }^{1-4}$ The Disability Adjusted Life Years (DALY) rate for cervical cancer in 2016 was highest in Karnataka, followed by Tamil Nadu, Chhattisgarh, Madhya Pradesh, and Maharashtra. Cervical cancer was the second leading cause of cancer deaths for females in 12 Indian states. ${ }^{1,3-5}$

The estimated risk of cervical cancer is calculated out to be approximately 1 in 53 Indian women during their lifetime compared with 1 in 100 women in developed nations. ${ }^{6-8}$ In India, cervical cancer is the most common cancer accounting for $25.9 \%$ of new cancer cases and $23.3 \%$ of all cancer related deaths. ${ }^{9-11}$ Statistically, yearly approximately 90,000 new cases of cervical cancer are noted. The incidence in India is 45 per one lakh women. ${ }^{3,8,9,11}$ The disease is more prevalent in people living in poor living conditions and low-income groups. Prevention of cervical cancer is regarded as a very sensitive and important public health issue.

Widespread screening of women with the Papanicolaou test has led to a substantial decrease in incidence and mortality due to cervical cancer in countries where it has been systematically implemented. ${ }^{10,11}$

During the recent decades consensus has been reached on the direct relationship between HPV infection and cervical carcinogenesis, ${ }^{14,15}$ and it has been demonstrated that HPV infection is a necessary condition for the development of pre-invasive and invasive cervical

\footnotetext{
${ }^{*}$ Corresponding author.

E-mail addresses: drcskapoor@yahoo.co.in (C.S. Kapoor), manishabhardwaj17@gmail.com (M. Sharma).
} 
cancer. ${ }^{12,13}$ Currently more than 200 HPV types have been identified and those infecting the cervix have been categorized according to their oncogenic potential as high-risk (HR). As HR-HPV types are detected in more than $99 \%$ of invasive cancer cases and in the vast majority of high-grade pre-invasive cases, HPV detection may be a reasonable alternative as a screening test for the detection of precursor lesions that would progress to cancer if not treated. In fact, the detection of HR-HPV types is considered today as a better method of screening than cytology. (7).

Due to lack of data on cervical cancer prevalence in regions near to Delhi NCR, this study would give a prevalence rate and incidence rate of HPV infection leading to cervix cancer. There are some reports that are available for HPV prevalence in Delhi region but no data available for its adjacent cities like Gurgaon (Haryana). Rural NCR has barriers to accessing screening services including cultural constraints/beliefs about the illness, economic factors, domestic gender power relations, alternative authoritative sources of reproductive health knowledge and unfriendly health care services. ${ }^{26}$

Considering the challenges faced in accessing cervical cancer screening to introduce importance of vaccination in rural areas, it is important to understand the uptake of these services and factors that affect their utilisation so as to inform effective interventions. This study assessed uptake of cervical cancer screening and associated factors among women in rural NCR region.

\section{Material and methods}

\subsection{Study population}

Women aged 20-60 years who had indications of cervical malignancy and those that attended the gynaecology and cancer OPD in a tertiary care center/hospital of Haryana, India.

\subsection{Tools}

A pretested questionnaire was prepared that had critical parameters listed down for effective study and analysis. These questionnaires were asked to fill by the study participants and included questions on: Age and Marital status; Age of marriage and Parity; ever screened for cervical cancer; When was the last screening test done; Year completed in formal education; Hormonal contraceptives use; What are the abnormalities you are facing and since when; Tobacco/smoking use; Sanitation; Economic status.

\subsection{Techniques}

Pap smear; visual inspection with acetic acid; clinical biopsies (in consultation with gynaecologist); DNA isolation from biopsy samples (phenol/chloroform method); HPV serotyping (PCR primer specific).

\subsection{Study design}

A prospective observational descriptive study. A prior permission from the Institutional Ethical committee was obtained. This study did not impose financial burden on study participants. A well-informed consent in writing was received from the study subjects before conducting the study. All participants were ensured confidentiality. The study mainly included a descriptive survey. In this design, a questionnaire was prepared, pretested and asked to the patients coming to gynaecologist and consulting cancer department. All the information collected was kept confidential. After screening, the diagnosis reports were collected from doctors, e.g. Pap smear or inspection visually with acetic acid. Simultaneously HPV serotyping was done by PCR under laboratory controls. After the disease diagnosis, information was collected on treatment strategies.
Table 1

Distribution of HPV prevalence by age, age at marriage \& at first pregnancy of the participants.

\begin{tabular}{lcll}
\hline Age (in years) & Total subjects & N (\%) & P-value \\
\hline $20-30$ & 62 & $30(48.4)$ & $0.0001^{*}$ \\
$30-40$ & 72 & $41(56.9)$ & \\
$40-50$ & 70 & $64(91.4)$ & \\
$>50$ & 16 & $16(100.0)$ & \\
Age at marriage (in years) & 194 & $138(71.1)$ & $0.01^{*}$ \\
$11-18$ & 26 & $13(50.0)$ & \\
$18-22$ & 178 & $126(70.7)$ & $0.04^{*}$ \\
Age at 1st pregnancy (in years) & $25(59.5)$ & \\
$13-18$ & 42 & & \\
$19-23$ & & & \\
\hline
\end{tabular}

*Statistically significant.

\subsection{Statistical analysis}

Data entry, data cleaning \& data analyses were done by Statistical Package for Social Sciences software (version 20.0; SPSS). Output indicators were analysed through the software and results were subsequently presented in form of tables \& graph. Mean (SD; standard deviation) were calculated for continuous variables and proportions for categorical variables. Proportions were compared using chi-square whereas means were compared using independent $t$-test. For all comparisons, the p-value of $<0.05$ was considered statistically significant. Sample size of 196 was calculated based on assuming the prevalence of HPV infection to be $60 \%$, absolute precision of $7 \%$, and $90 \%$ power. We further assumed a non-response rate of $10 \%$. Hence, the total sample size was 220 . We used the formula $4 \mathrm{PQ} / \mathrm{d}^{2}$ where $\mathrm{P}=$ prevalence; $\mathrm{Q}=100-\mathrm{P} ; \mathrm{d}=$ absolute precision.

\section{Results}

The mean (SD) age of the participants was 37.8 (9.2) years and most of the study participants were aged between 11 and 18 years at the time of their marriage (88.2\%) (Table 1). Mean (SD) age at the time of first pregnancy among the study subjects was 16.8 (2.7) years. Only around $19 \%$ were aged between 19 and 23 years at the time of their first pregnancy (Table 1). The mean (SD) age of the participants at the time of their first PAP smear testing was 37.6 (9.0) years. A total of $29.1 \%$ participants had their first PAP smear between the age range of 20-30 years; $32.7 \%$ in the age group of $30-40$ years; $31.8 \%$ in the age range of 40-50 years (Table 1). All the study subjects were married $(100.0 \%)$ and had a parity of 1-3 (51.8\%) followed by those with the parity of 4 or more $(46.4 \%)$. All of the study participants were not vaccinated with HPV vaccine before screening. Among the study subjects, women who were having tobacco or were smoking, had HPV prevalence of around 98.4\%; and for those who were not having any of these had HPV prevalence reduced to $31.6 \%$ (Table 2$)(\mathrm{P}=0.0001)$. Among the study subjects, women who below poverty line had HPV prevalence of $72.2 \%$; for subjects whose socioeconomic status was average, HPV prevalence was reduced to $42.3 \%$ and for those whose socioeconomic status was good, they did not had HPV. This shows that with increasing socioeconomic status HPV prevalence reduces $(\mathrm{P}=0.002)$ [Table 2]. Among the study subjects, women with good sanitation had HPV prevalence of $25 \%$ and those with poor sanitation had HPV prevalence of $69.4 \%$ $(\mathrm{P}=0.02)$ [Table 2]. Majority of the study participants had not heard about PAP testing $(93.6 \%)$ and did not understand the importance of PAP testing (92.7\%).

Only a small proportion $(0.9 \%)$ reported use of contraception. Similar proportions of the study subjects had either no abortions (49.1\%) or between 1 and 2 abortions (49.1\%) (Table 3). Majority of the study participants complained of pain, white discharge, pain while intercourse and itching (81.4\%) (Table 3). A total of $68.6 \%$ of the study subjects had an HPV infection. Among those that had an HPV infection, 
Table 2

Distribution of HPV prevalence by education status, parity, sanitation level and tobacco/smoking habit.

\begin{tabular}{llll}
\hline No. of parity & Total subjects & N (\%) & P-value \\
\hline 0 & 4 & $1(25.0)$ & $0.0001^{*}$ \\
$1-3$ & 114 & $60(52.6)$ & \\
$\geq 4$ & 102 & $90(88.2)$ & \\
Sanitation level & & $1(25.0)$ & $0.02^{*}$ \\
Good & 4 & $150(69.4)$ & \\
Poor & 216 & $120(98.4)$ & $0.0001^{*}$ \\
Tobacco/smoking habit & 122 & $31(31.6)$ & \\
Yes & 98 & $140(72.2)$ & $0.002^{*}$ \\
No & & $11(42.3)$ & \\
Socioeconomic status & 194 & $0(0.0)$ & \\
Below poverty line & 26 & & \\
Average & 0 & & \\
Good & & & \\
\hline
\end{tabular}

*Statistically significant.

Table 3

Distribution of study participants according to use of contraceptives, or have knowledge/understanding of PAP smear, types of HPV infection and presenting features reported.

\begin{tabular}{ll}
\hline Number of abortions & $\mathrm{N}(\%)$ \\
\hline 0 & $108(49.1)$ \\
$1-2$ & $108(49.1)$ \\
$\geq 3$ & $4(1.8)$ \\
Use of contraceptives & \\
Yes & $2(0.9)$ \\
No & $218(99.1)$ \\
Hemorrhage & $160(72.7)$ \\
Nes & $60(27.3)$ \\
HPV infection & $151(68.6)$ \\
Yes & $69(31.4)$ \\
No & \\
HPV serotype & $144(95.4)$ \\
HPV 16 & $7(4.6)$ \\
HPV 18 & \\
Genital warts concurrence & $160(72.7)$ \\
Present & $60(27.3)$ \\
Absent & \\
Pain, white discharge, Pain while intercourse, Itching, warts (before & $179(81.4)$ \\
$\quad$ menopause) & $41(18.6)$ \\
Menopause &
\end{tabular}

majority had HPV 16 infection (95.4\%) while in the remaining 4.6\%, HPV 18 was present (Table 3). Majority of the study participants had presence of genital warts concurrently (72.7\%). On PAP smear, Dense inflammation was present in around two-third (63.6\%) of the participants. Squamous cell NILM in the magnitude of $70 \%$ was present in $25.5 \%$ participants; in the magnitude of $75 \%$ was present in $7.2 \%$ and in the magnitude of $80 \%$ was present in only $1.8 \%$ participants (Table 3). Findings show that with increasing age HPV prevalence increases (Table 1). We observed various factors like early age at marriage, lack of education, increased parity, early age at first pregnancy, poor sanitation, use of tobacco and belonging to below poverty line were significantly associated with HPV infection.

\section{Discussion}

The present study collected demographic, epidemiologic and clinical information of women of aged group 20-60 years with gynaecological abnormalities who attended the gynaecology and cancer OPD. A cross sectional study was conducted in Delhi region among 1411 women aged below 35 years revealed that $36.3 \%$ of women who had reported earlier gynaecological problems and merely around a third of the participants (30.8\%) were aware about cervical cancer in general. ${ }^{17}$ A quantitative study in Malaysia showed that $48.7 \%$ woman were aware of cervical cancer and screening. ${ }^{18}$ Similarly, an exploratory study was conducted among 356 women at Zimbabwe revealed that only $9.8 \%$ of women knew about pap smear and $12.6 \%$ knew about visual inspection using acetic acid (VIA). ${ }^{19}$ Similarly, in our study, we observed that knowledge and understanding for Pap smear among studied women were seen in merely $5-6 \%$ cases and rest women were not aware of this test for cervical cancer screening. World Health Organization has concluded that screening for cervical cancer should be performed at least once for every woman in the target age group (30-49 years) when it is most beneficial. The recommended screening tests includes: HPV testing, cytology and visual inspection with acetic acid (VIA). The valuable holistic target approaches should be either "screenand-treat" or "screen, diagnose and treat". ${ }^{20,21}$

In a cross-sectional study among 524 Indian women with symptoms of persistent vaginal discharge, intermenstrual bleeding, and postcoital bleeding, a wide spectrum of HPV infection has been seen in north Indian women, with the majority being HPV-16 type seen in $74.3 \%$ cases. $^{22}$ In our study, a total of $68.6 \%$ of the study subjects those that had an HPV infection, out of which 95\% had HPV-16 infection.

The primary cause of pre-cancerous and cancerous cervical lesions is infection with a high-risk or oncogenic HPV types. ${ }^{23}$ The majority of cervical cancer cases occur as a result of HPV 16 and 18 infection. Among which, HPV 16 are found to be highly prevalent in high risk cases. This is well known that infection is predominantly transmitted by sexual contact, causing mostly squamous type intraepithelial lesions. ${ }^{23,24}$ However, due to body's immunological response, most lesions disappear after 6-12 months. However, a small percentage of these lesions remain and can cause cancer. ${ }^{25}$ The results of a metaanalysis showed that the highest prevalence of HPV occurs at the age of 25 years which could be related to changes in sexual behaviour. In another meta-analysis study, the bimodal distribution of cervical cancer in some regions has been studied i.e. age at first sexual intercourse, a sudden spurt (first peak) in HPV can be observed, followed by a plateau stage at adult age; then, after 45 years of age, the second peak again is noticed. In a developing country like India, carcinoma of cervix is seen at the prime age of 47 years. $^{1-5}$ The mean age of carcinoma in-situ (CIN) is 28 years. ${ }^{1,3}$ However, carcinoma of cervix is frequently seen in 4th and 5th decades of life. The disease can occur in both extremes of life. In our study, the mean age of the participants was 38 years.

Permanent infection with one of the high-risk types of HPV over time leads to the development of cervical intraepithelial neoplasia (CIN). The major mechanisms through which HPV contributes to carcinogenesis involve the activity of two viral oncoproteins, E6 and E7, which interfere with major tumor suppressor genes, P53 and retinoblastoma. In addition, E6 and E7 are associated with changes in host DNA and virus DNA methylation that are associated with changes in key cellular pathways that regulate genetic integrity, cell adhesion, immune response, apoptosis, and cellular control. ${ }^{26}$

An increased risk of cervical cancer is observed in people with multiple sexual partners This increase in risk is generally attributable to an increased risk of HPV infection. Also, early age at first intercourse is a risk factor for cervical cancer. We observed this age to be as low as 16.8 years in our study cohort.

Several studies have shown that full-term pregnancy increases the risk of invasive cervical cancer. Moreover, high parity increases this risk of cancer in women. In a collaborative international epidemiological study of cervical cancer, a direct correlation was found between the risk of cervical cancer and parity; there was an inverse association between the risk of cervical cancer and the age of the mother at first pregnancy. The risk of cervical cancer significantly increases with an increase in the number of deliveries. In a cohort study with over 13 years of follow-up, delivery was the predictor of CIN 3 especially among women with high risk HPV infection. In our study, HPV prevalence was reported to be highest (90\%) among women with parity $\geq 4$. 
The current and recent use of combined oral contraceptive (OC) methods is associated with an increased risk of cervical cancer. This was in contrast with findings of our study where this was found in less than $1 \%$ cases because of unawareness among rural study participants. Based on the results of an international epidemiological study of cervical cancer, the relative risk is found to be increased with prolonged duration of OC use. It has been reported that the use of OC method for 5 years or more can double the risk of cancer.

To date, various studies have shown that smoking is one of the most important risk factors for carcinoma in situ stage: 3 and invasive cervical cancer. We observed direct association of tobacco and smoking with HPV prevalence in $98 \%$ cases. This has been observed in a large cohort study that the risk of cancer was decreased by one half among those who ceased smoking for 10 years compared to those who remain smoker. Moreover, the duration of smoking is coherent to the increased risk of cervical cancer. This study has some limitations, as this study was conducted among women from rural background and hence the generalizability of the findings across India may not be scientific. Study was concentrated more on quantitative aspects by using the close ended questions in a small cohort of patients.

\section{Conclusion and recommendations}

The study reported a high prevalence of HPV infection in women attending the Gynaecology and Cancer OPD of a tertiary care hospital. Several important risk factors for the HPV infection were also identified. These were early age at marriage, lack of education, increased parity, early age at first pregnancy, poor sanitation, use of tobacco and belonging to below poverty line. These findings call for more efforts to increase awareness pertaining to HPV infection and cervical cancer among women. Health education, barrier-specific counselling and outreach and community-based interventions should be ideal to provide an opportunity and to enhance the awareness of having cervical screening done.

\section{Acknowledgements}

I would like to give my sincere gratitude to my supervisor Dr. Chandrashekhar Kapoor for providing their guidance and suggestions during my project. Also all the doctors and nursing staff for helping me during patients data collection.

\section{References}

1. Denny L. Cervical cancer: prevention and treatment. Discov Med. 2012;14:125-131.

2. Satija A. Cervical cancer in India. South Asia centre for chronic disease. . Available from: http://sancd.org/uploads/pdf/cervical_cancer.pdf, Accessed date: 16 February 2014.

3. Arbyn M, Castellsague X, DeSanjose S, et al. Worldwide burden of cervical cancer. Ann Oncol. 2011;22:2675-2686.
4. Yeole BB, Kumar AV, Kurkureet A, Sunny L. Population-based survival from cancers of breast, cervix and ovary in women in Mumbai. Asian Pac J Cancer Prev APJCP. 2004;5:308-315.

5. ICO Information Centre on HPV and cancer. Human Papillomavirus and Related Diseases in India Summary Report 2014-08-22 2014; 2014 ([Google Scholar]).

6. National Cancer Control Programme. . Available from: http://www.mohfw.nic.in/ showfile.php?lid=324, Accessed date: 16 July 2014 .

7. National Programme for Prevention and Control of Cancer. Diabetes, cardiovascular disease and stroke. . Available from: http://www.nrhmhp.gov.in/sites/default/files/ files/NCD_Guidelines.pdf, Accessed date: 15 July 2014.

8. Gakidou E, Stella N, Ziad O. Coverage of cervical cancer screening in 57 countries: low average levels and large inequalities. PLoS Med. 2009;5:e132.

9. Aswathy S, Quereshi MA, Kurian B, Leelamoni K. Cervical cancer screening: current knowledge and practice among women in a rural population of Kerala, India. Indian $J$ Med Res. 2012;136(2):205-210.

10. Quinn M, Babb P, Jones J, Allen E. Effect of screening on incidence of and mortality from cancer of cervix in England: evaluation based on routinely collected statistics. BMJ. 1999;318:904-908 PMID:10102852.

11. Jemal A, Ward E, Thun M. Declining death rates reflect progress against cancer. PLoS One. 2010;5:e9584https://doi.org/10.1371/journal.pone.0009584 PMID: 20231893.

12. Bosch FX, de Sanjose S. Chapter 1: human papillomavirus and cervical cancerburden and assessment of causality. J Natl Cancer Inst. 2003 Monogr: 3-13.

13. Bulkmans NW, Rozendaal L, Snijders PJ, et al. POBASCAM, a population-based randomized controlled trial for implementation of high-risk HPV testing in cervical screening: design, methods and baseline data of 44,102 women. Int J Cancer. 2004;110:94-101 PMID: 15054873.

14. Zur Hausen H. Human papillomaviruses and their possible role in squamous cell carcinomas. Curr Top Microbiol Immunol. 1977;78:1-30 PMID: 202434.

15. Walboomers JM, Jacobs MV, Manos MM, et al. Human papillomavirus is a necessary cause of invasive cervical cancer worldwide. J Pathol. 1999;189:12-19 PMID: 10451482 .

17. Ortashi O, Raheel H, Shalal M, Osman N. Awareness and knowledge about human papillomavirus infection and vaccination among women in UAE. Asian Pac J Cancer Prev APJCP. 2013;14(10):6077-6080.

18. Sabeena S, Bhat PV, Kamath V, Aswathyraj S, Arunkumar G. Knowledge, attitude and practice concerning human papilloma virus infection and its health effects among rural women, Karnataka, South India. Asian Pac J Cancer Prev APJCP. 2015;16(12):5053-5058.

19. Kulkarni SS, Kulkarni SS, Vastrad PP, et al. Prevalence and distribution of high risk human papillomavirus (HPV) Types 16 and 18 in Carcinoma of cervix, saliva of patients with oral squamous cell carcinoma and in the general population in Karnataka, India. Asian Pac J Cancer Prev APJCP. 2011;12(3):645-648.

20. Human Papilloma Virus ICMR. High Power Committee to Evaluate Performance of ICMR, 2012-2013. New Delhi, India: ICMR; 2014 (Disease Specific Documents for XII plan).

21. Bansal AB, Pakhare AP, Kapoor N, Mehrotra R, Kokane AM. Knowledge, attitude, and practices related to cervical cancer among adult women: a hospital-based crosssectional study. J Nat Sci Biol Med. 2015 Dec;6(2):324-328.

22. Sharma J, Day P, Puroshottam R. Current understanding on HPV infection and cervical cancer along with risk factor among women of urban Delhi. Gynecol Oncol. 2013;118:S12-S17.

23. De Sanjose, Laimins L. A meta-analytic study on pathogenesis of human papillomaviruses. Microbiol Mol Biol. 2004;68:362-372.

24. Liu S, Chan K, Leung R, et al. Meta-analysis on the prevalence and risk factors of Human Papillomavirus (HPV) infection. PLoS One. 2013;6:e19244.

25. Zhao FH, Lewkowitz AK, Hu SY, et al. Prevalence of human papillomavirus and cervical intraepithelial neoplasia in China: a pooled analysis of 17 population-based studies. Int J Cancer. 2012;131:2929-2938.

26. Sudenga SL, Rositch AF, Otieno WA, Smith JS. Knowledge, attitudes, practices, and perceived risk of cervical cancer among Kenyan women: brief report. Epub 2013/ 05/23 Int J Gynecol Cancer: Off J Int Gynecol Canc Soc. 2013;23(5):895-899. 